\title{
Calculation of the Ostriker-Vishniac effect in cold dark matter models
}

\author{
Andrew H. Jaffe* \\ Center for Particle Astrophysics, 301 LeConte Hall, University of California, Berkeley, California 94720 \\ Marc Kamionkowski ${ }^{\dagger}$ \\ Department of Physics, Columbia University, 538 West 120th Street, New York, New York 10027
}

(Received 6 January 1998; published 10 July 1998)

\begin{abstract}
We present a new derivation of the cosmic microwave background anisotropy spectrum from the OstrikerVishniac effect for an open, flat, or closed universe, and calculate the anisotropy expected in cold dark matter (CDM) models. We provide simple semi-analytic fitting formulas for the Vishniac power spectrum that can be used to evaluate the expected anisotropy in CDM models for any arbitrary ionization history. In a flat universe, $\mathrm{CDM}$ models normalized to cluster abundances produce rms temperature anisotropies of $0.8-2.4 \mu \mathrm{K}$ on arcminute angular scales for a constant ionization fraction of unity, whereas an ionization fraction of 0.2 yields rms anisotropies of $0.3-0.8 \mu \mathrm{K}$. In an open and/or high-baryon-density universe, the level of anisotropy is somewhat higher. The signal in some of these models may be detectable with planned interferometry experiments. The damping of the acoustic peaks in the primary-anisotropy spectrum at degree angular scales depends primarily on the optical depth and only secondarily on the epoch of reionization. On the other hand, the amplitude of Ostriker-Vishniac anisotropies depends sensitively on the epoch of reionization. Therefore, when combined with the estimate of the reionization optical depth provided by maps of degree-scale anisotropies, the Ostriker-Vishniac effect can provide a unique probe of the epoch of reionization. [S0556-2821(98)03816-8]
\end{abstract}

PACS number(s): 98.70.Vc, 98.80.Cq

\section{INTRODUCTION}

Standard cold dark matter (CDM) and its variants are the current leading models for the origin of large-scale structure. The canonical CDM model hypothesizes a primordial scalefree spectrum of primordial adiabatic perturbations and a critical density of cold dark matter. Its variants include lowdensity (e.g., $\Omega_{0} \simeq 0.3-0.4$ ) models, either in an open universe or in a flat universe with a cosmological constant, tilted models in which the power-law index $n$ of the primordial power spectrum differs slightly from scale-free $(n=1)$, low-Hubble-constant models, or mixed-dark-matter models in which the Universe has a critical density but roughly $30 \%$ is in the form of hot dark matter.

Although most of the matter in these models does not undergo gravitational collapse until relatively late in the history of the universe, some small fraction of the mass is expected to collapse at early times. Ultraviolet photons released by this early generation of star and/or galaxy formation will partially reionize the Universe, and these ionized electrons will re-scatter at least some cosmic microwave background (CMB) photons after recombination at a redshift of $z$ $\simeq 1100$. Theoretical uncertainties in the process of star formation and the resulting ionization make precise predictions of the ionization history difficult. Constraints to the shape of the CMB blackbody spectrum and detection of CMB anisotropy at degree angular scales suggest that if reionization occurred, the fraction of CMB photons that re-scattered is small. Still, estimates show that even if small, at least some reionization is expected in CDM models [1-3]: for example, the most careful recent calculations suggest a fraction

\footnotetext{
*Email address: jaffe@ physics.berkeley.edu

${ }^{\dagger}$ Email address: kamion@ phys.columbia.edu
}

$\tau_{r} \sim 0.1$ of CMB photons were re-scattered [3].

Scattering of CMB photons from ionized clouds will lead to anisotropies at arcminute separations below the Silkdamping scale (the Ostriker-Vishniac effect) [4-10]. These anisotropies arise at a higher order in perturbation theory and are therefore not included in the usual Boltzmann calculations of CMB anisotropy spectra. The level of anisotropy is expected to be small and it has so far eluded detection. However, these anisotropies may be observable with forthcoming CMB interferometry experiments [11] that probe the CMB power spectrum at arcminute scales.

In this paper, we present a new derivation of the anisotropy spectrum from the Ostriker-Vishniac effect. We then calculate the anisotropy expected in CDM models. We provide numerical results for the anisotropy predicted by a variety of CDM models with several plausible ionization histories. We also provide simple semi-analytic fitting formulas for the Ostriker-Vishniac power spectrum that can be used to evaluate the expected anisotropy in CDM models for any arbitrary ionization history. We estimate the anisotropy amplitude that may be detectable with forthcoming interferometry experiments. We find that the signal should be detectable in a number of CDM models with plausible ionization histories.

To a good approximation, the damping of the acoustic peaks in the primary (linear-theory) CMB spectrum from reionization is the same for any ionization history that produces the same optical depth to the surface of last scatter (see, e.g., Ref. [1]). Therefore, although MAP and Planck [12] should provide a good estimate of this optical depth [13], they will not strongly constrain the ionization history, that is, whether reionization occurred earlier or later. On the other hand, our results indicate that the amplitude of the Ostriker-Vishniac anisotropy depends primarily on the ionization fraction and only secondarily on the optical depth. 
Therefore, when combined with the determination of the optical depth from MAP and Planck, the Ostriker-Vishniac effect can determine the epoch of reionization. This will be important for precise determination of the baryon density, Hubble constant, and cosmological constant from the higher acoustic peaks in the CMB spectrum. It will also provide a unique window to the epoch at which structures first undergo gravitational collapse in the Universe.

\section{PRELIMINARIES}

The scale factor of the Universe, $a(t)$, satisfies the Friedmann equations,

$$
\begin{gathered}
\frac{\dot{a}}{a}=H_{0} E(z) \\
\equiv H_{0} \sqrt{\Omega_{0}(1+z)^{3}+\Omega_{\Lambda}+\left(1-\Omega_{0}-\Omega_{\Lambda}\right)(1+z)^{2}}, \\
\frac{\ddot{a}}{a}=H_{0}^{2}\left[\Omega_{\Lambda}-\Omega_{0}(1+z)^{3} / 2\right],
\end{gathered}
$$

where $H_{0}=100 \mathrm{hm} \mathrm{sec}^{-1} \mathrm{Mpc}^{-1}$ is the Hubble constant, $\Omega_{0}$ is the current nonrelativistic-matter density in units of the critical density, $\Omega_{\Lambda}$ is the current contribution of the cosmological constant to the critical density, and the overdot denotes a derivative with respect to time. If $\Omega_{0}+\Omega_{\Lambda}=1$, the Universe is flat; if it is greater (less) than unity the Universe is closed (open). An Einstein-de Sitter universe has $\Omega_{0}=1$ and $\Omega_{\Lambda}=0$, so $E(z)=(1+z)^{3 / 2}$.

We choose the scale factor such that $a_{0} H_{0}=2$. If we are located at the origin, $\mathbf{w}=0$, then an object at redshift $z$ is at a comoving distance,

$$
w(z)=\frac{1}{2} \int_{0}^{z} \frac{d z^{\prime}}{E\left(z^{\prime}\right)} .
$$

We define a conformal time by $d \eta=d t / a$, so the comoving distance to the horizon is the conformal time today, $\eta_{0}$ $=w(\infty)$. In an Einstein-de Sitter universe, $a / a_{0}=\eta^{2}$ and $\eta_{0}=1$ with our conventions.

If the density contrast at comoving position $\mathbf{w}$ at time $t$ is $\delta(\mathbf{w}, t)$, with Fourier transform

$$
\begin{aligned}
& \widetilde{\delta}(\mathbf{k})=\int d^{3} \mathbf{w} \exp (-i \mathbf{k} \cdot \mathbf{w}) \delta(\mathbf{w}), \\
& \delta(\mathbf{w})=\int \frac{d^{3} \mathbf{k}}{(2 \pi)^{3}} \exp (i \mathbf{k} \cdot \mathbf{w}) \widetilde{\delta}(\mathbf{k}),
\end{aligned}
$$

then the power spectrum $P(k, t)$ is defined by the expectation value

$$
\left\langle\widetilde{\delta}(\mathbf{k}, t) \widetilde{\delta}^{*}\left(\mathbf{k}^{\prime}, t\right)\right\rangle=(2 \pi)^{3} \delta\left(\mathbf{k}-\mathbf{k}^{\prime}\right) P(k, t),
$$

where the angle brackets denote an average over all realizations. In linear theory, the spatial and time dependence of $\delta$ can be factorized, so $\delta(\mathbf{w}, t)=\delta_{0}(\mathbf{w}) D(t) / D\left(t_{0}\right)$, where $t_{0}$ is the age of the Universe, $\delta_{0}(\mathbf{w})=\delta\left(\mathbf{w}, t_{0}\right)$, and the growth factor (written as a function of redshift) is [14]

$$
D(z)=\frac{5 \Omega_{0} E(z)}{2} \int_{z}^{\infty} \frac{1+z^{\prime}}{\left[E\left(z^{\prime}\right)\right]^{3}} d z^{\prime} .
$$

The time $t$ is related to the redshift by

$$
t(z)=\frac{1}{H_{0}} \int_{z}^{\infty} \frac{d z^{\prime}}{\left(1+z^{\prime}\right) E\left(z^{\prime}\right)} .
$$

Therefore, the power spectrum is $P(k, t)=P(k, 0)\left(D / D_{0}\right)^{2}$, where $D_{0} \equiv D\left(t_{0}\right)$. In an Einstein-de Sitter universe, $a / a_{0}$ $=\left(t / t_{0}\right)^{2 / 3}$, and $D(t) / D_{0}=a(t) / a_{0}$.

In linear theory, the Fourier components of the velocity field are related to those of the density field by

$$
\widetilde{\mathbf{v}}(\mathbf{k}, t)=\frac{i a(t)}{k^{2}} \frac{\dot{D}}{D} \mathbf{k} \widetilde{\delta}(\mathbf{k}, t)=\frac{i a(t)}{k^{2}} \frac{\dot{D}}{D_{0}} \mathbf{k} \widetilde{\delta}_{0}(\mathbf{k}) .
$$

It can also be shown [14] that

$$
\frac{\dot{D}}{D}=\frac{\ddot{a}}{\dot{a}}-\frac{\dot{a}}{a}+\frac{5 \Omega_{0}}{2} \frac{\dot{a}}{a} \frac{(1+z)^{2}}{[E(z)]^{2} D(z)} .
$$

\section{THE OSTRIKER-VISHNIAC EFFECT}

The Ostriker-Vishniac effect is the CMB anisotropy produced by scattering from ionized regions or clouds with bulk peculiar velocities. The fractional temperature perturbation in the direction $\vec{\theta}$ induced by bulk motions is

$$
p(\vec{\theta}) \equiv \frac{\Delta T}{T}(\vec{\theta})=-\int_{0}^{\eta_{0}} n_{e} \sigma_{T} e^{-\tau}[\hat{\theta} \cdot \mathbf{v}(w \hat{\theta} ; w) / c] a(w) d w,
$$

where $n_{e}$ is the electron density along the line of sight, $\mathbf{v}(\mathbf{w} ; w)$ is the bulk velocity at position $\mathbf{w}$ at a conformal time $\eta_{0}-w, \sigma_{T}$ is the Thomson cross section, and $\tau$ is the optical depth from us to $w$. The factor of $a(x)$ arises because the physical time is $d t=a(x) d x$. [Note that $\hat{\theta}$ represents a threedimensional unit vector along the line of sight, whereas $\vec{\theta}$ will refer to a dimensionless two-dimensional vector in the plane perpendicular to the line of sight-i.e., for directions near $\hat{\mathbf{z}}, \hat{\theta}=\left(\theta_{1}, \theta_{2}, \sqrt{1-\theta_{1}^{2}-\theta_{2}^{2}}\right) \simeq\left(\theta_{1}, \theta_{2}, 1\right)$, whereas $\vec{\theta}=\left(\theta_{1}, \theta_{2}, 0\right)$.] The visibility function,

$$
g(w)=\overline{n_{e}}(w) \sigma_{T} a(w) e^{-\tau(w)}=(d \tau / d w) e^{-\tau}
$$

is the probability distribution for scattering from reionized electrons. Here, $\overline{n_{e}}(w)=\Omega_{b} \rho_{c} x_{e}(w)(1+z)^{3} / m_{p}$ is the mean electron density, where $\Omega_{b}$ is the baryon density today in units of the critical density (actually, this number should be multiplied by $7 / 8$ to account for the neutrons bound in he- 
lium nuclei); $\rho_{c}$ is the critical density; $x_{e}(w)$ is the ionization fraction; and $m_{p}$ is the proton mass. The visibility function is normalized so that

$$
\int_{0}^{\infty} g(w) d w=1-e^{-\tau_{r}}
$$

where $\tau_{r}$ is the optical depth to the standard-recombination surface of the scatter, the fraction of CMB photons which scattered after standard recombination at $z \simeq 1100$.

With these developments, the fractional temperature perturbation becomes

$$
p(\vec{\theta})=-\int_{0}^{\eta_{0}} d w g(w) \hat{\theta} \cdot \mathbf{q}(w \hat{\boldsymbol{\theta}}, w),
$$

where $\mathbf{q}(\mathbf{w}, w)=[1+\delta(\mathbf{w}, w)] \mathbf{v}(\mathbf{w}, w)$. According to Eq. (13), only the component of $\mathbf{v}$ along the line of sight contributes to the anisotropy. In linear theory, we neglect $\delta \ll 1$ and the total anisotropy is the sum of the contributions of each Fourier component of the velocity field. However, in linear theory (and in fact to all orders), $\widetilde{\mathbf{v}}(\mathbf{k}) \propto \mathbf{k}$, so only $\mathbf{k}$ modes along the line of sight can contribute to the anisotropy. The contributions of troughs and crests of each Fourier mode cancel approximately when projected along the line of sight. Therefore, the contribution of bulk velocities to anisotropies vanishes (or is very small) to lowest order in perturbation theory on small angular scales. On larger angular scales, there are fewer crests and troughs, so the cancellation is not as complete.

In other words, angular correlations (on small angular scales) can be due only to $\mathbf{k}$ modes perpendicular to the line of sight, and these contribute nothing to the anisotropy from bulk velocities alone. Furthermore, no anisotropy can be produced by $\mathbf{q}$ modes parallel to $\mathbf{k}$, and therefore the anisotropy can be due only to the components $\widetilde{\mathbf{q}}_{\perp}(\mathbf{k})$ perpendicular to $\mathbf{k}$ $[4,5,7,8]$.

In the Appendix, we make this argument more precise and show that the projection, Eq. (13), has Fourier coefficients that satisfy

$$
\left\langle\tilde{p}(\vec{\kappa}) \tilde{p}^{*}\left(\vec{\kappa}^{\prime}\right)\right\rangle=(2 \pi)^{2} \delta\left(\vec{\kappa}-\vec{\kappa}^{\prime}\right) P_{p}(\kappa),
$$

with

$$
P_{p}(\kappa)=\frac{1}{2} \int_{0}^{\eta_{0}} \frac{g^{2}(w)}{w^{2}} P_{\perp}(\kappa / w, w) d w,
$$

in a flat universe [making the replacement Eq. (A10) for a closed or open universe] where $P_{\perp}(k, w)$ is the threedimensional power spectrum for $\tilde{\mathbf{q}}_{\perp}(\mathbf{k})$. We now need to determine this three-dimensional power spectrum.

Again, $\tilde{\mathbf{v}}(\mathbf{k}) \propto \mathbf{k}$, so $\tilde{\mathbf{q}}_{\perp}(\mathbf{k})$ can only come from the Fourier transform of the nonlinear term $\delta(\mathbf{w}) \mathbf{v}(\mathbf{w})$. The Fourier transform of $\delta(\mathbf{w}, t) \mathbf{v}(\mathbf{w}, t)$ [not the full $(1+\delta) \mathbf{v}]$ is

$$
\begin{aligned}
\widetilde{\mathbf{q}}(\mathbf{k}, t) & =\frac{1}{2} \int \frac{d^{3} \mathbf{k}^{\prime}}{(2 \pi)^{3}}\left[\widetilde{\delta}\left(\mathbf{k}^{\prime}, t\right) \widetilde{\mathbf{v}}\left(\mathbf{k}-\mathbf{k}^{\prime}, t\right)+\widetilde{\delta}\left(\mathbf{k}-\mathbf{k}^{\prime}, t\right) \widetilde{\mathbf{v}}\left(\mathbf{k}^{\prime}, t\right)\right] \\
& =\frac{i a \dot{D} D}{2 D_{0}^{2}} \int \frac{d^{3} \mathbf{k}^{\prime}}{(2 \pi)^{3}} \widetilde{\delta}_{0}\left(\mathbf{k}^{\prime}\right) \widetilde{\delta}_{0}\left(\mathbf{k}-\mathbf{k}^{\prime}\right)\left(\frac{\mathbf{k}-\mathbf{k}^{\prime}}{\left|\mathbf{k}-\mathbf{k}^{\prime}\right|^{2}}+\frac{\mathbf{k}^{\prime}}{k^{\prime 2}}\right)
\end{aligned}
$$

The components of $\tilde{\mathbf{q}}$ perpendicular to $\mathbf{k}$ are

$$
\begin{aligned}
q_{\perp, i}(\mathbf{k}, t) & =\left(\delta_{i j}-\frac{k_{i} k_{j}}{k^{2}}\right) q_{j}(\mathbf{k}, t) \\
& =\frac{i a \dot{D} D}{2 D_{0}^{2}} \int \frac{d^{3} \mathbf{k}^{\prime}}{(2 \pi)^{3}} \widetilde{\delta}_{0}\left(\mathbf{k}^{\prime}\right) \widetilde{\delta}_{0}\left(\mathbf{k}-\mathbf{k}^{\prime}\right)\left(k_{i}^{\prime}-\frac{k_{i}\left(\mathbf{k} \cdot \mathbf{k}^{\prime}\right)}{k^{2}}\right)\left(\frac{1}{k^{\prime 2}}-\frac{1}{\left|\mathbf{k}-\mathbf{k}^{\prime}\right|^{2}}\right) .
\end{aligned}
$$

For a Gaussian density field,

$$
\begin{aligned}
\left\langle\widetilde{\delta}_{0}\left(\mathbf{k}_{1}-\mathbf{k}^{\prime}\right) \widetilde{\delta}_{0}\left(\mathbf{k}^{\prime}\right) \widetilde{\delta}_{0}^{*}\left(\mathbf{k}_{2}-\mathbf{k}^{\prime \prime}\right) \widetilde{\delta}_{0}^{*}\left(\mathbf{k}^{\prime \prime}\right)\right\rangle & \\
& =(2 \pi)^{6} P\left(\left|\mathbf{k}_{1}-\mathbf{k}^{\prime}\right|, t\right) P\left(k^{\prime}, t\right)\left[\delta\left(\mathbf{k}_{1}-\mathbf{k}_{2}\right) \delta\left(\mathbf{k}^{\prime}-\mathbf{k}^{\prime \prime}\right)+\delta\left(\mathbf{k}_{1}-\mathbf{k}_{2}\right) \delta\left(\mathbf{k}_{1}-\mathbf{k}^{\prime}-\mathbf{k}^{\prime \prime}\right)\right] .
\end{aligned}
$$


We combine these expressions to find (after some extensive although straightforward algebra)

$$
\left\langle\tilde{\mathbf{q}}_{\perp}(\mathbf{k}, t) \cdot \tilde{\mathbf{q}}_{\perp}^{*}\left(\mathbf{k}^{\prime}, t\right)\right\rangle=(2 \pi)^{3} \delta\left(\mathbf{k}-\mathbf{k}^{\prime}\right) P_{\perp}(\mathbf{k}, t),
$$

with

$$
P_{\perp}(k, w)=\frac{a^{2}(w)}{8 \pi^{2}}\left(\frac{\dot{D} D}{D_{0}^{2}}\right)^{2} S(k) ;
$$

the Vishniac power spectrum is [5]

$$
\begin{aligned}
S(k)= & k \int_{0}^{\infty} d y \int_{-1}^{1} d x P(k y) P\left(k \sqrt{1+y^{2}-2 x y}\right) \\
& \times \frac{\left(1-x^{2}\right)(1-2 x y)^{2}}{\left(1+y^{2}-2 x y\right)^{2}} .
\end{aligned}
$$

[The differences with Eq. (2.13) in [5] are the result of differing Fourier conventions.]

Assembling these results, in a flat universe, the OstrikerVishniac effect produces a power spectrum,

$$
P_{p}(\kappa)=\frac{1}{16 \pi^{2}} \int_{0}^{\eta_{0}} \frac{g^{2}(w)}{w^{2}}[a(w)]^{2}\left(\frac{\dot{D} D}{D_{0}^{2}}\right)^{2} S(\kappa / w) d w,
$$

with the Vishniac power spectrum $S(k)$ given by Eq. (21). In an open or closed universe, one makes the replacement Eq. (A10) in the argument of $S(\kappa / w)$ and in the two factors of $w$ in the denominator of the integrand the numbers for the open models in the table and the open-model curves need to be fixed.

\section{A. Gaussian visibility function}

If the visibility function is approximated as a Gaussian in conformal time,

$$
g(w)=\frac{1-e^{-\tau_{r}}}{\sqrt{2 \pi(\Delta w)^{2}}} \exp \left[-\frac{1}{2} \frac{\left(w-w_{r}\right)^{2}}{(\Delta w)^{2}}\right],
$$

where $w_{r}$ is the comoving distance to the re-scattering surface, $\Delta w$ is the width of the re-scattering surface, and $\tau_{r}$ is the fraction of CMB photons re-scattered, then the CMB power spectrum is

$$
P_{p}(\kappa) \simeq \frac{\left(1-e^{\left.-\tau_{r}\right)^{2}}\right.}{32 \pi^{2} \sqrt{\pi}(\Delta w) w_{r}^{2}}\left[\frac{a\left(w_{r}\right) \dot{D}\left(w_{r}\right) D\left(w_{r}\right)}{D_{0}^{2}}\right]^{2} S\left(\kappa / w_{r}\right) .
$$

In practice, the visibility functions for the ionization histories usually considered are very poorly approximated by a Gaussian. Still, the Gaussian approximation provides a simple analytic description of how the anisotropy spectrum depends on the redshift of re-scattering, optical depth, and $S(k)$.

\section{B. Relation to multipole moments}

Given the power spectrum $P_{p}(\kappa)$ and our Fourier conventions, it is straightforward to write the temperature autocorrelation function in terms of the power spectrum:

$$
C(\alpha)=\langle p(\vec{\theta}+\vec{\alpha}) p(\vec{\theta})\rangle=\frac{1}{2 \pi} \int \kappa d \kappa J_{0}(\kappa \alpha) P_{p}(\kappa) .
$$

The correlation function can also be written in terms of the more commonly used CMB multipole moments $C_{\ell}$ as

$$
C(\alpha)=\sum_{\ell} \frac{2 \ell+1}{4 \pi} P \ell(\cos \alpha) C_{\ell},
$$

where $P(\cos \alpha)$ are Legendre polynomials. Since $P \ell(\cos \theta) \rightarrow J_{0}(\ell \theta)$ as $\ell \rightarrow \infty$, it follows that

$$
C_{\ell}=P_{p}(\kappa=\ell) \text {. }
$$

This identification allows us to illustrate our results in the multipole moments that have become familiar and to compare our results with those of previous authors.

\section{Comparison with previous results}

First, we compare our results with the results of Ref. [7] (and therefore Ref. [6]). To do so, we consider only the Einstein-de Sitter universe as they do. In an Einstein-de Sitter universe, Eq. (22) becomes (after changing the integration variable to $\left.\eta=\eta_{0}-w\right)$,

$$
P_{p}(\kappa)=\frac{1}{4 \pi^{2}} \int_{0}^{\eta_{0}} \frac{g^{2}\left(w=\eta_{0}-\eta\right)}{\left(\eta_{0}-\eta\right)^{2} \eta_{0}^{2}}\left(\frac{\eta}{\eta_{0}}\right)^{6} S\left(\frac{\kappa}{\eta_{0}-\eta}\right) d \eta .
$$

We then note that they approximate the power spectrum by evaluating $S(\kappa / w)$ at $w=\eta_{0}$ and taking it outside the integral in Eq. (28). Now we compare our result with Eq. (6.15f) in Ref. [7] and their $J$ in their Eq. (6.18). If we note that their $K(k) P^{2}(k)$ is equal to our $S(k) / k$ and assume that $\eta_{0}-\eta$ $\simeq \eta_{0}$ in the integrand, then our results reproduce theirs (although it appears that their result is twice ours, we have not been able to locate the source of the discrepancy).

Now we want to compare our results with [10]. Although their derivation, unlike ours, relies on a solution to the Boltzmann equation, our results should agree. Specifically, our Eq. (22) should agree with their Eq. (24). To check that this is so, we change our integration variable in Eq. (22) to $k$ $=\ell / w$ and find

$$
C_{\ell}=\int_{\eta_{0}}^{\infty} d k W_{\ell}(k) S(k)
$$

where

$$
W_{\ell}(k)=\frac{1}{16 \pi^{2} \ell}[g(w=\ell / k)]^{2}\left(\frac{a \dot{D} D}{D_{0}^{2}}\right)^{2} .
$$


If we put their Eq. (24) into this form [to do this, keep in mind that their overdot denotes differentiation with respect to $\eta$ instead of $t$, so our $a \dot{D}$ is equal to their $\dot{D}$-also, they normalize $D$ such that $D\left(t_{0}\right)=1$ ], we find agreement with their results.

\section{Variance of temperature distribution}

The variance of the temperature distribution induced by the Ostriker-Vishniac effect is

$$
\left\langle(\Delta T / T)^{2}\right\rangle=\frac{1}{2 \pi} \int \ell d \ell C_{\ell} .
$$

In the Gaussian approximation for $g(w)$ and in a flat universe,

$$
\begin{aligned}
\left\langle(\Delta T / T)^{2}\right\rangle= & \frac{\tau_{r}^{2}}{32 \pi^{3} \sqrt{\pi}(\Delta w)}\left[\frac{a\left(w_{r}\right) \dot{D}\left(w_{r}\right) D\left(w_{r}\right)}{D_{0}^{2}}\right]^{2} \\
& \times \int k d k S(k),
\end{aligned}
$$

and for an Einstein-de Sitter universe this simplifies to

$$
\left\langle(\Delta T / T)^{2}\right\rangle=\frac{\tau_{r}^{2}}{32 \pi^{3} \sqrt{\pi}(\Delta w)} \frac{4}{\eta_{0}^{2}}\left(\frac{\eta_{r}}{\eta_{0}}\right)^{6} \int k d k S(k) .
$$

\section{THE SPATIAL POWER SPECTRUM}

For the power spectrum, we use

$$
P(k)=\frac{2 \pi^{2}}{8} \delta_{H}^{2}(k / 2)^{n} T^{2}\left(k_{p} \mathrm{Mpc} / h \Gamma\right),
$$

where $T(q)$ is the usual CDM transfer function, $k_{p}=k a_{0}$ $=k H_{0} / 2$ is the physical wave number with our conventions, and $\Gamma \simeq \Omega_{0} h$ is given more accurately in terms of $\Omega_{0} h$ and the baryon fraction $\Omega_{b}$ by Eqs. (D-28) and (E-12) in Ref. [15]. The factor of 8 in the denominator in Eq. (34) arises because we are using $a_{0} H_{0}=2$. For the transfer function, we use [16]

$$
T(q)=\frac{\ln (1+2.34 q) /(2.34 q)}{\left[1+3.89 q+(16.1 q)^{2}+(5.46 q)^{3}+(6.71 q)^{4}\right]^{1 / 4}} .
$$

For $\delta_{H}$, we use the fits to the $C O B E$ anisotropy given in [17]. For the tilted model, we use the $C O B E$ normalization from Ref. [17] obtained including tensor modes with the amplitude predicted by power-law inflation. (Note that the tensor contribution in other inflationary models may be different.)

Alternatively, the power spectrum may be normalized at small distance scales through the cluster abundance which fixes $\sigma_{8}$, the variance in the mass enclosed in spheres of radius $8 h^{-1} \mathrm{Mpc}$, to $\sigma_{8} \simeq(0.6 \pm 0.1) \Omega_{0}^{-0.53}$ [18]. In terms of the power spectrum,

$$
\sigma_{8}^{2}=\frac{1}{2 \pi^{2}} \int k^{2} d k P(k)\left[\frac{3 j_{1}\left(k_{p} R\right)}{k_{p} R}\right]^{2},
$$

where $R=8 h^{-1} \mathrm{Mpc}$, and $j_{1}(x)$ is a spherical Bessel function. Since we are using $a_{0} \neq 1, k_{p}$ (rather than $k$ ) enters into the argument of the spherical Bessel function.

\section{NUMERICAL RESULTS}

In this section we present numerical results for the anisotropy spectrum and rms temperature fluctuation for a variety of CDM models and ionization histories. We consider a family of ionization histories parametrized by a constant ionization fraction $x_{e}$ after a redshift $z_{r}$. In a cosmologicalconstant universe, this induces an optical depth,

$$
\begin{aligned}
\tau_{r} & =\left(\Omega_{b} \rho_{c} \sigma_{T} a_{0} / m_{p}\right) \int_{0}^{z_{r}} \frac{(1+z)^{2} d z}{E(z)} \\
& =0.069 \Omega_{b} h x_{e} \int_{0}^{z_{r}} \frac{(1+z)^{2} d z}{E(z)} .
\end{aligned}
$$

In a flat universe, this becomes

$$
\tau_{r}=0.046 \Omega_{b} h x_{e}\left[\sqrt{\Omega_{0}\left(1+z_{r}\right)^{3}+\left(1-\Omega_{0}\right)}-1\right] .
$$

The prefactor 0.069 (or 0.046 in the flat-universe expression) is obtained if all the baryons are in the form of protons, an assumption often made when calculating ionization histories. If one takes into account the fact that one quarter of the baryonic mass is helium, then the prefactor should be multiplied by $7 / 8$.

Table I lists the predicted rms temperature anisotropy $\left\langle(\Delta T)^{2}\right\rangle^{1 / 2}$ due to the Ostriker-Vishniac effect for a variety of CDM models, which are parametrized by $\Omega_{0}, \Omega_{\Lambda}, h, n$, and $\Omega_{b} h^{2}$, and ionization histories, which are parametrized by $x_{e}$ and $z_{r}$. The table also lists $\tau_{r}, \sigma_{8}$, and $\sigma_{8} \Omega_{0}^{0.53}$. We present a variety of models and ionization histories to illustrate the effects of variation of various parameters on the predictions. It should be kept in mind that not all of the $C O B E$-normalized models satisfy the aforementioned observational constraint to $\sigma_{8}$ from cluster abundances [18]. We therefore list in the table the predicted rms temperature anisotropy expected if the $C O B E$ normalization is disregarded and the amplitudes of the power spectrum is fixed instead to the cluster abundance. Similarly, the $h=0.35$ model is meant to be illustrative; this value of the Hubble constant is in disagreement with most measurements.

Figure 1 shows the predicted anisotropy for the canonical standard-CDM model $\left(\Omega_{0}=1, \Omega_{\Lambda}=0, h=0.5, n=1, \Omega_{b} h^{2}\right.$ $=0.0125)$, for a variety of ionization histories, with the linear power spectrum, also shown, normalized to COBE. We know from quasar absorption spectra that the Universe has been significantly reionized at least since a redshift of $z_{r}$ $=5$. Therefore, we include predictions for this minimal level of anisotropy.

The effect of reionization on the primary anisotropies can be quantified primarily by the optical depth $\tau_{r}$ [1]; any two 
TABLE I. The rms temperature perturbation $\left\langle(\Delta T / T)^{2}\right\rangle^{1 / 2}$ in $\mu \mathrm{K}$ due to the Ostriker-Vishniac effect and value of $\ell_{\text {peak }}$ at which $\ell(\ell+1) C /$ peaks for a variety of CDM models and ionization histories for models normalized to both $C O B E$ and cluster abundances.

\begin{tabular}{|c|c|c|c|c|c|c|c|c|c|c|c|c|}
\hline$\Omega_{0}$ & $\Omega_{\Lambda}$ & $h$ & $n$ & $\Omega_{b} h^{2}$ & $\sigma_{8}$ & $\sigma_{8} \Omega_{0}^{0.53}$ & $z_{r}$ & $x_{e}$ & $\tau_{r}$ & $(\Delta T)_{\mathrm{rms}}^{\mathrm{COBE}}$ & $(\Delta T)_{\mathrm{rms}}^{\text {cluster }}$ & $\ell_{\text {peak }}$ \\
\hline 1 & 0 & 0.5 & 1 & 0.0125 & 1.21 & 1.21 & 5 & 1.0 & 0.016 & 3.1 & 0.77 & 4000 \\
\hline 1 & 0 & 0.5 & 1 & 0.0125 & 1.21 & 1.21 & 19 & 1.0 & 0.1 & 4.8 & 1.2 & 6300 \\
\hline 1 & 0 & 0.5 & 1 & 0.0125 & 1.21 & 1.21 & 56 & 0.2 & 0.1 & 1.4 & 0.34 & 7900 \\
\hline 1 & 0 & 0.5 & 1 & 0.0125 & 1.21 & 1.21 & 56 & 1.0 & 0.5 & 6.2 & 1.5 & 7900 \\
\hline 1 & 0 & 0.5 & 1 & 0.0125 & 1.21 & 1.21 & 166 & 0.2 & 0.5 & 1.7 & 0.42 & 7900 \\
\hline 1 & 0 & 0.5 & 0.8 & 0.025 & 0.53 & 0.53 & 12 & 1.0 & 0.1 & 1.2 & 1.6 & 3200 \\
\hline 1 & 0 & 0.5 & 0.8 & 0.025 & 0.53 & 0.53 & 35 & 0.2 & 0.1 & 0.37 & 0.48 & 4000 \\
\hline 1 & 0 & 0.5 & 0.8 & 0.025 & 0.53 & 0.53 & 35 & 1.0 & 0.5 & 1.6 & 2.1 & 4000 \\
\hline 1 & 0 & 0.5 & 0.8 & 0.025 & 0.53 & 0.53 & 104 & 0.2 & 0.5 & 0.46 & 0.60 & 4000 \\
\hline 0.4 & 0.6 & 0.65 & 1 & 0.015 & 1.07 & 0.65 & 27 & 1.0 & 0.1 & 2.5 & 2.1 & 4000 \\
\hline 0.4 & 0.6 & 0.65 & 1 & 0.015 & 1.07 & 0.65 & 81 & 0.2 & 0.1 & 0.73 & 0.62 & 5000 \\
\hline 0.4 & 0.6 & 0.65 & 1 & 0.015 & 1.07 & 0.65 & 81 & 1.0 & 0.5 & 3.3 & 2.8 & 5000 \\
\hline 0.4 & 0.6 & 0.65 & 1 & 0.015 & 1.07 & 0.65 & 238 & 0.2 & 0.5 & 0.92 & 0.77 & 6300 \\
\hline 1 & 0 & 0.35 & 1 & 0.015 & 0.74 & 0.74 & 13 & 1.0 & 0.1 & 2.4 & 1.6 & 4000 \\
\hline 1 & 0 & 0.35 & 1 & 0.015 & 0.74 & 0.74 & 39 & 0.2 & 0.1 & 0.70 & 0.46 & 5000 \\
\hline 1 & 0 & 0.35 & 1 & 0.015 & 0.74 & 0.74 & 39 & 1.0 & 0.5 & 3.1 & 2.1 & 4000 \\
\hline 1 & 0 & 0.35 & 1 & 0.015 & 0.74 & 0.74 & 116 & 0.2 & 0.5 & 0.87 & 0.57 & 5000 \\
\hline 0.4 & 0 & 0.8 & 1 & 0.0125 & 0.87 & 0.54 & 19 & 1.0 & 0.1 & 2.3 & 2.8 & 7900 \\
\hline 0.4 & 0 & 0.8 & 1 & 0.0125 & 0.87 & 0.54 & 54 & 0.2 & 0.1 & 0.79 & 0.99 & 10000 \\
\hline 0.4 & 0 & 0.8 & 1 & 0.0125 & 0.87 & 0.54 & 54 & 1.0 & 0.5 & 3.2 & 4.0 & 10000 \\
\hline 0.4 & 0 & 0.8 & 1 & 0.0125 & 0.87 & 0.54 & 156 & 0.2 & 0.5 & 1.0 & 1.3 & 10000 \\
\hline 0.3 & 0 & 0.7 & 1 & 0.05 & 0.30 & 0.16 & 7 & 1.0 & 0.1 & 0.79 & 11 & 2500 \\
\hline 0.3 & 0 & 0.7 & 1 & 0.05 & 0.30 & 0.16 & 20 & 0.2 & 0.1 & 0.31 & 4.4 & 4000 \\
\hline 0.3 & 0 & 0.7 & 1 & 0.05 & 0.30 & 0.16 & 20 & 1.0 & 0.5 & 1.3 & 19 & 3100 \\
\hline 0.3 & 0 & 0.7 & 1 & 0.05 & 0.30 & 0.16 & 57 & 0.2 & 0.5 & 0.44 & 6.4 & 5000 \\
\hline
\end{tabular}

ionization histories that have the same $\tau_{r}$ produce roughly the same primary anisotropies. However, as Fig. 1 and Table I show, the secondary anisotropies are more dependent on the ionization fraction than on the optical depth.

The growth of density perturbations leads to a growth of the peculiar velocities that induce the secondary anisotropy. Therefore, if reionization takes place later, the anisotropy is significantly larger, even for the same optical depth. Furthermore, if reionization takes place later, the peak of the anisotropy spectrum, which is fixed in comoving distance scale by the peak of the power spectrum, subtends a larger angle. In open models, the peak of the power spectrum moves to larger physical scales, while the effects of geometry shift these physical scales back to smaller angular scales; the result is that the spectrum peaks at only slightly lower $\ell$ than in a flat universe.

The weakness of the dependence of the anisotropy amplitude on $\tau_{r}$ indicates that a good fraction of the anisotropy is produced at later times when the amplitude of the power spectrum has grown. The convergence of the predictions at small $\ell$ for ionization histories with the same $x_{e}$ indicates that, in particular, the anisotropy at larger scales comes primarily from lower redshifts [10], and therefore that assuming that re-scattering occurs primarily at the epoch of reionization $[6,7]$ does not provide a good approximation, especially at larger scales. Moreover, in open models the evolution of 


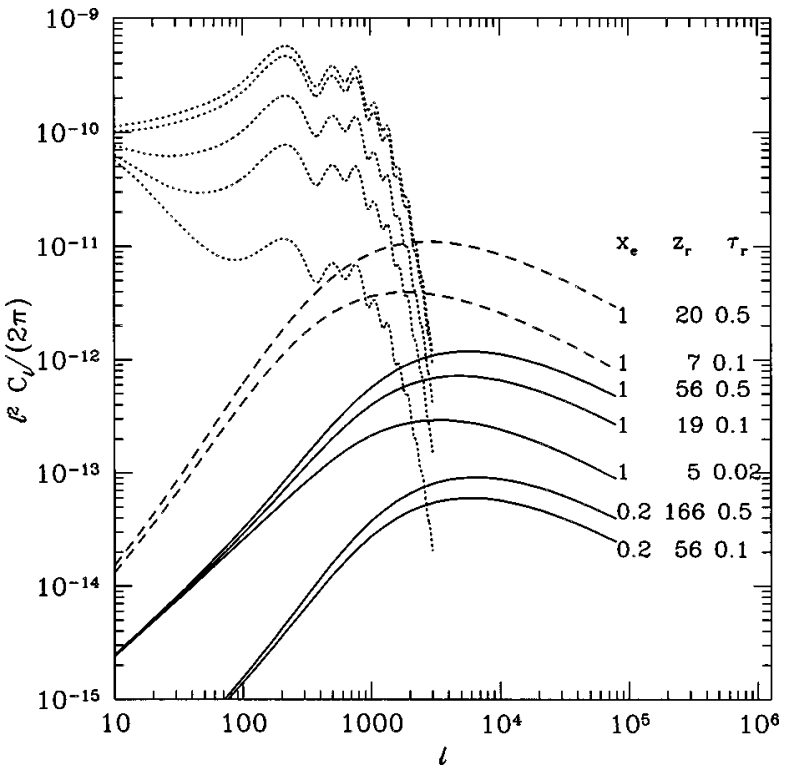

FIG. 1. Multipole moments for the Ostriker-Vishniac effect for the $C O B E$-normalized canonical standard-CDM model $(\Omega=1, h$ $\left.=0.5, n=1, \Omega_{b} h^{2}=0.0125\right)$, for a variety of ionization histories, as listed. We also show predictions for several open high-baryondensity models with the same $x_{e}$ and $\tau_{r}$, normalized to the cluster abundance, with dashed curves. The dotted curves show the primary anisotropy for this model for $\tau_{r}=0.0,0.1,0.5,1$, and 2 , from top to bottom.

the growth factor differs from a flat universe by larger amounts at later times; therefore the slope of $C_{\ell}$ at larger scales is greater than in a flat universe.

The growth of density perturbations induces an initially Gaussian distribution of perturbations to become nonGaussian. Therefore, the distribution of anisotropies produced by the Ostriker-Vishniac effect will be non-Gaussian even for Gaussian initial conditions, and the higher $n$-pt temperature correlation functions may be calculated in perturbation theory [19].

The anisotropy is due primarily to density perturbations on distance scales closer to those probed by galaxy surveys than to those probed by $C O B E$. Furthermore, the anisotropy is due to a large extent to scattering at later times. Therefore, when the power spectra are normalized to the cluster abundance, the spread in predicted values for the rms anisotropy is smaller than it is if the models are normalized to COBE. Since the anisotropy is a second-order effect, the rms anisotropy is proportional to $\sigma_{8}^{2}$. In a flat universe, models normalized to cluster abundances produce rms temperature anisotropies, listed in Table I, of $0.8-3 \mu \mathrm{K}$ for a constant ionization fraction of unity, whereas an ionization fraction of 0.2 yields rms anisotropies of $0.3-0.8 \mu \mathrm{K}$.

Our numerical results can also be easily scaled for a different value of the ionization fraction $x_{e}$ if the ionization epoch is held fixed and if $\tau_{r} \ll 1$. The rms temperature anisotropy should be roughly proportional to $x_{e}$ if $z_{r}$ is held fixed. Also, if we neglect the effect of $\Omega_{b}$ and $h$ on the power spectrum (in particular, $\Omega_{b}$ should have only a relatively weak effect on the power spectrum as long as $\Omega_{b} \ll \Omega_{0}$ ), then
Eq. (37) shows that the rms anisotropy should be proportional to the combination $\Omega_{b} h x_{e}=\left(\Omega_{b} h^{2}\right) x_{e} / h$, itself proportional to the column density of electrons to the last scattering surface. This also suggests that the Ostriker-Vishniac anisotropy in models with a high baryon density should be larger. To illustrate (and to illustrate the effects of geometry), we also include in Table I and in Fig. 1 predictions for an open model with $\Omega_{b} \simeq \Omega_{0} / 3$, as suggested possibly by a peak in the observed power spectrum at $100 h^{-1} \mathrm{Mpc}$ [20]. Highbaryon-density models will be explored more carefully in Ref. [21].

\section{APPROXIMATE VISHNIAC POWER SPECTRA FOR CDM MODELS}

Here we provide simple approximate analytic fits to the Vishniac power spectrum $S(k)$ for standard-CDM power spectra. These can be used to quickly estimate the OstrikerVishniac anisotropy for CDM models with an arbitrary ionization history.

With the standard-CDM power spectrum given in the form of Eq. (34), $S(k)$ may be written,

$$
S(k)=\frac{\pi^{4} \delta_{H}^{4}}{16}(k / 2)^{2 n} k I_{n}\left(k_{p} \mathrm{Mpc} / h \Gamma\right),
$$

where

$$
\begin{aligned}
I_{n}(q)= & \int_{0}^{\infty} d y \int_{-1}^{1} d x\left(y \sqrt{1+y^{2}-2 x y}\right)^{n} T^{2}(y q) T^{2} \\
& \times\left(q \sqrt{1+y^{2}-2 x y}\right) \frac{\left(1-x^{2}\right)(1-2 x y)^{2}}{\left(1+y^{2}-2 x y\right)^{2}} .
\end{aligned}
$$

For the standard-CDM transfer function, Eq. (35), the functions $I_{n}(q)$ may be approximated, for $0.5 \lesssim n \lesssim 1$, by an asymmetric Gaussian:

$$
I_{n}(q) \simeq \frac{h(n)}{4(q / 2)^{2 n+3}} \exp \left\{-\frac{1}{2}\left[\frac{\log _{10}[q]-\log _{10}\left[q_{0}(n)\right]}{\sigma(n)}\right]^{2}\right\},
$$

with $\log _{10}[h(n)]=-4.716-0.636(n-1)+0.832(n-1)^{2}$, $\log _{10}\left[q_{0}(n)\right]=0.477+1.36(n-1)+0.975(n-1)^{2}, \quad$ and $\sigma$ $=0.6+0.33(n-1)+0.34(n-1)^{2}$ for $q<q_{0}$, and $\sigma=0.87$ $+1.105(n-1)+1.19(n-1)^{2}$ for $q>q_{0}$.

Figure 2 shows the multipole moments obtained from numerical calculation of $S(k)$ and the analytic approximation given here for a variety of models. Although this analytic approximation does not accurately reproduce the asymptotic large- and small- $k$ behavior of $S(k)$, it accurately approximates the peak of $k^{2} S(k)$. As indicated by Eq. (32), this should provide reliable estimates of the mean-square anisotropy.

\section{DISCUSSION}

Although precise limits depend on imprecisely determined cosmological parameters, the existence of significant 

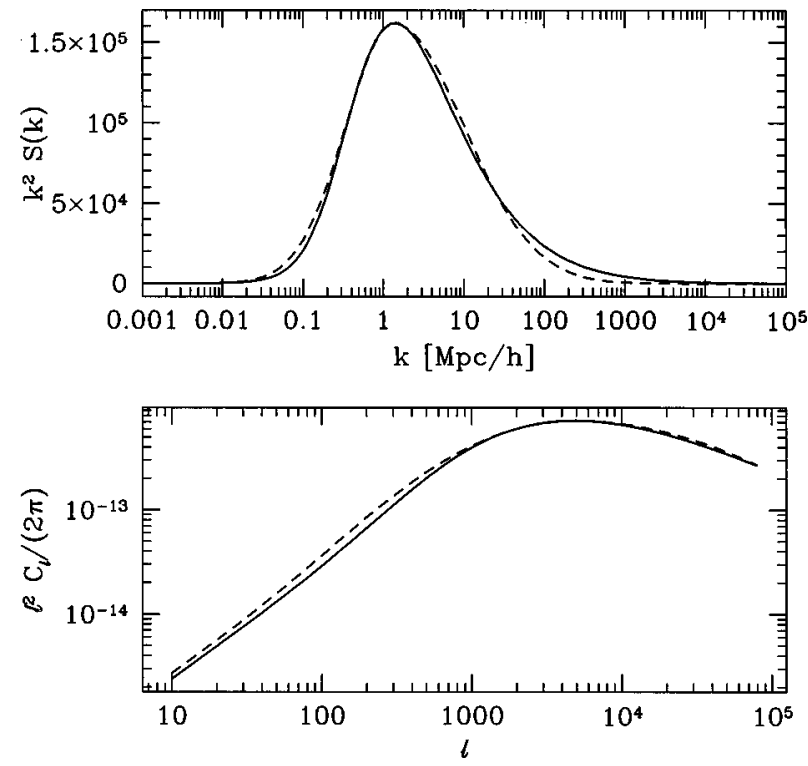

FIG. 2. Semi-analytic fits to $S(k)$, the Vishniac power spectrum. Top: full calculation (solid) and the analytic approximation (dashed) for the standard-CDM $S(k)$. Bottom: $\ell(\ell+1) C /(2 \pi)$ calculated with the full $S(k)$ (solid) and the approximation (dashed) for standard CDM with an ionization history given by $z_{r}=19, x_{e}$ $=1.0, \tau_{r}=0.1$.

CMB anisotropy at the degree scale and current limits to arcminute-scale anisotropy and distortions to the CMB blackbody spectrum seem to indicate that $\tau_{r} \leqslant 1$. However, some reionization is generically expected in CDM models. Again, the detailed ionization history will depend on the model and on details of star formation that are still difficult to quantify reliably. Still, optical depths $\tau_{r}=\mathcal{O}(0.1)$ are observationally consistent and expected in CDM models.

Such a level of reionization will lead to secondary anisotropies at arcminute scales like those shown in Sec. V. Furthermore, the bulk of the reionization in CDM models generally occurs later, so the secondary anisotropy will be larger for a given $\tau_{r}$ than if reionization occurred earlier. The predicted level of anisotropy in virtually all of the models we considered here is smaller than the current limit of about 50 $\mu \mathrm{K}$ at arcminute angular separations [22]. However, there has been much recent progress in the design of interferometer experiments dedicated to CMB measurements, and there should be much more in forthcoming years. To estimate the signal that might be detectable, we suppose that a detector with a sensitivity $s$ (in $\mu \mathrm{K} \sqrt{\mathrm{sec}}$ ) maps a fraction $f_{\text {sky }}$ of the sky with an angular resolution $\theta_{\text {fwhm }} \leqslant 1^{\prime}$ sufficient to detect these anisotropies for a time $t_{\text {mon }}$ months. Such a detector should be sensitive to an rms temperature anisotropy as small as

$$
(\Delta T)_{\mathrm{rms}}^{\min } \simeq 0.5 \mu \mathrm{K} \frac{(s / 1000 \mu \mathrm{K} \sqrt{\mathrm{sec}}) f_{\mathrm{sky}}^{1 / 4}}{\left(\theta_{\mathrm{fwhm}} / 0.1^{\circ}\right)^{1 / 2} t_{\mathrm{mon}}^{1 / 2}} .
$$

Although the interferometry experiments that will likely reach these angular scales are not really parametrized by a sensitivity $s$, the effective value of $s$ for these experiments is already better than $1000 \mu \mathrm{K} \sqrt{\mathrm{sec}}$. Furthermore, with a full power-spectrum analysis of the data, the sensitivity could be improved perhaps by an order of magnitude over this simple estimate. Comparing Eq. (42) for a reasonable $t_{\text {mon }}$ with the predictions in Sec. V, it seems quite plausible-even though the experiments may be challenging-that the OstrikerVishniac anisotropy expected in many leading CDM models will be detectable. The results of Ref. [11] imply that the anisotropy generated by some of the more optimistic models considered here may already be detectable by upcoming experiments such as CBI, especially if binned over a wide range of $\ell$.

Observation of the Ostriker-Vishniac effect will be important for understanding the ionization history of the Universe and for determining cosmological parameters with the CMB. The linear-theory anisotropies depend primarily on the optical depth $\tau_{r}$ and only secondarily on the details of the ionization history (e.g., the values of $z_{r}$ and $x_{e}$ ). Therefore, degree-scale anisotropies may determine $\tau_{r}$, but they will not strongly constrain the epoch of reionization. When combined with sensitive measurements of the degree-scale anisotropy, measurement of the Ostriker-Vishniac effect will therefore provide the epoch of reionization.

The shape of the CMB power spectrum for these anisotropies is relatively featureless. Therefore, even detection of the anisotropy, without a precise determination of the shape of the power spectrum, will provide the most essential information on the ionization history. Of course, more precise constraints to the ionization history can be obtained if the power spectrum can be mapped and, some shape information along with multi-frequency observations will be necessary to distinguish the Ostriker-Vishniac anisotropy from foreground contamination such as that induced by extragalactic point sources.

Determination of the geometry of the Universe relies primarily on the first acoustic peak in the primary CMB spectrum $[1,23]$. However, precise and reliable determination of other cosmological parameters, such as the baryon density, Hubble constant, cosmological constant, and spectral index, depends on the structure of the smaller-scale acoustic peaks. The details of the ionization history (e.g., the epoch of reionization, which we have explored here) will, in fact, have some effect on these higher peaks. This will in turn affect the reconstruction of some cosmological parameters, although this uncertainty has not yet been taken into account in the parameter-estimation analyses of Ref. [13]. Therefore, the information on the epoch of reionization provided by mapping the secondary anisotropies will be important for precise determination of these other cosmological parameters with MAP and Planck.

It seems unlikely that the current FIRAS constraints to Compton- $y$ distortions of the CMB blackbody spectrum will be improved significantly. Therefore, the Ostriker-Vishniac effect seems to be the most promising probe of the ionization history. In addition to its significance for cosmologicalparameter determination, the Ostriker-Vishniac effect can provide a window to the epoch of the earliest collapsed objects that is inaccessible with any other observations. 


\section{ACKNOWLEDGMENTS}

We thank M. White and W. Hu for useful comments. This work was supported at Columbia by D.O.E. contract No. DEFG02-92-ER 40699, NASA NAG5-3091, and the Alfred P. Sloan Foundation and at Berkeley by NAG5-6552. M.K. would like to acknowledge the hospitality of the Center for Particle Astrophysics where part of this work was completed.

\section{APPENDIX: PROJECTED POWER SPECTRUM FOR A DIVERGENCE-FREE VECTOR FIELD}

If an isotropic random field $\mathbf{q}(\mathbf{w})$ is decomposed into Fourier components,

$$
\widetilde{\mathbf{q}}(\mathbf{k})=\int d^{3} \mathbf{w} \exp (-i \mathbf{k} \cdot \mathbf{w}) \mathbf{q}(\mathbf{w})
$$

then the Fourier components may be written as the sum of divergence-free and curl-free parts parts: $\tilde{\mathbf{q}}(\mathbf{k})=\widetilde{\mathbf{q}}_{\perp}(\mathbf{k})$ $+\widetilde{\mathbf{q}}_{\|}(\mathbf{k})$ with $\mathbf{k} \cdot \tilde{\mathbf{q}}_{\perp}(\mathbf{k})=0$ and $\mathbf{k} \times \widetilde{\mathbf{q}}_{\|}(\mathbf{k})=0$; in particular, $\mathbf{q}_{\|}(\mathbf{k})=\hat{\mathbf{k}} q_{\|}(\mathbf{k})$.

Suppose we observe a projection

$$
p(\vec{\theta})=-\int_{0}^{\eta_{0}} d w g(w) \hat{\theta} \cdot \mathbf{q}(w \hat{\theta}, w ; w),
$$

of $\hat{\theta} \cdot \mathbf{q}(w \hat{\boldsymbol{\theta}}, w)$, the component of $\mathbf{q}(\mathbf{w})$ along the line of sight, with a visibility function $g(w)$, and we define the Fourier components,

$$
\tilde{p}(\vec{\kappa})=\int d^{2} \vec{\theta} p(\vec{\theta}) e^{-i \vec{\kappa} \cdot \vec{\theta}}
$$

The contribution of the parallel components $\mathbf{q}(\mathbf{k})$ to $p(\vec{\theta})$ will be small because crests and troughs of each Fourier component projected along the line of sight will tend to cancel. The purpose of this appendix is to show that if the perpendicular components satisfy $\left\langle\tilde{\mathbf{q}}_{\perp}(\mathbf{k}) \cdot \tilde{\mathbf{q}}_{\perp}^{*}\left(\mathbf{k}^{\prime}\right)\right\rangle=(2 \pi)^{3} \delta(\mathbf{k}$ $\left.-\mathbf{k}^{\prime}\right) P_{\perp}(k)$, then the Fourier components of the projection satisfy

$$
\left\langle\tilde{p}(\vec{\kappa}) \tilde{p}^{*}\left(\vec{\kappa}^{\prime}\right)\right\rangle=(2 \pi)^{2} \delta\left(\vec{\kappa}-\vec{\kappa}^{\prime}\right) P_{p}(\kappa),
$$

with

$$
P_{p}(\kappa)=\frac{1}{2} \int_{0}^{\eta_{0}} d w \frac{g^{2}(w)}{w^{2}} P_{\perp}(\kappa / w, w) .
$$

To do so, we follow the steps in the Appendix of Ref. [24] that lead to the Fourier-space analogue of Limber's equation. Rather than reconstruct the entire argument, we refer the reader to Kaiser's paper [24] for more details.

The components of an isotropic divergence-free field satisfy [26],

$$
\left\langle\tilde{q}_{\perp, i}(\mathbf{k}) \tilde{q}_{\perp, j}^{*}\left(\mathbf{k}^{\prime}\right)\right\rangle=\frac{1}{2}\left(\delta_{i j}-\hat{\mathbf{k}}_{i} \hat{\mathbf{k}}_{j}\right)\left\langle\tilde{\mathbf{q}}_{\perp}(\mathbf{k}) \cdot \tilde{\mathbf{q}}_{\perp}^{*}\left(\mathbf{k}^{\prime}\right)\right\rangle .
$$

If the contribution of a narrow shell of width $\Delta w$ centered at $w_{0}$ is

$$
\Delta p(\vec{\theta})=g\left(w_{0}\right) \int_{w_{0}-\Delta w / 2}^{w_{0}+\Delta w / 2} d w \hat{\theta} \cdot \mathbf{q}_{\perp}\left(w_{0} \theta_{1}, w_{0} \theta_{2}, w ; w\right),
$$

then with Eq. (A6), we can show that its Fourier coefficients $\widetilde{\Delta p}(\vec{\kappa})$ satisfy

$$
\left\langle\widetilde{\Delta p}(\vec{\kappa}) \widetilde{\Delta p} *\left(\vec{\kappa}^{\prime}\right)\right\rangle=(2 \pi)^{2} \delta\left(\vec{\kappa}-\vec{\kappa}^{\prime}\right) \Delta P_{p}(\kappa),
$$

with

$$
\begin{aligned}
\Delta P_{p}(\kappa)= & \frac{1}{2} \frac{g^{2}\left(w_{0}\right) \Delta w}{w_{0}^{2}} \int \frac{d k_{3}}{2 \pi}\left[1-(\hat{\theta} \cdot \hat{k})^{2}\right] \\
& \times\left[j_{0}\left(k_{3} \Delta w / 2\right)\right]^{2} P_{\perp}\left(\sqrt{\frac{\kappa^{2}}{w_{0}^{2}}+k_{3}^{2}}\right),
\end{aligned}
$$

and $\mathbf{k}=\left(\kappa_{1} / w_{0}, \kappa_{2} / w_{0}, k_{3}\right)$. As argued by Kaiser, only modes with $k_{3} \ll \kappa / w_{0}$ (those very nearly perpendicular to the line of sight) will contribute appreciably to the integral, so $(\hat{\theta} \cdot \hat{k})^{2} \ll 1$. We then add the contributions $\widetilde{\Delta p}(\kappa)$ along the line of sight to obtain Eq. (A5).

In an open or closed universe one replaces

$$
w \rightarrow \frac{\mathcal{S}\left(a_{0} H_{0} w \sqrt{\left|1-\Omega_{0}-\Omega_{\Lambda}\right|}\right)}{a_{0} H_{0} \sqrt{\left|1-\Omega_{0}-\Omega_{\Lambda}\right|}}
$$

in the first argument of $P(\kappa / w, w)$ in Eq. (A5), and in the two factors of $w$ that appear in the denominator, where $\mathcal{S}(x)=\sinh x$ in an open universe and $\mathcal{S}(x)=\sin x$ in a closed universe [25]. ${ }^{1}$

Note that this derivation also explains why the contribution from $\mathbf{q}_{\|}$is suppressed. This term satisfies [26]

$$
\begin{aligned}
\left\langle\tilde{q}_{\|, i}(\mathbf{k}) \tilde{q}_{\|, j}^{*}\left(\mathbf{k}^{\prime}\right)\right\rangle & =\hat{k}_{i} \hat{k}_{j}\left\langle\tilde{q}_{\|}(\mathbf{k}) \tilde{q}_{\|}^{*}\left(\mathbf{k}^{\prime}\right)\right\rangle \\
& =(2 \pi)^{3} \delta\left(\mathbf{k}-\mathbf{k}^{\prime}\right) \hat{k}_{i} \hat{k}_{j} P_{\|}(k) .
\end{aligned}
$$

This would therefore have a contribution to the projected power spectrum of $\hat{\theta} \cdot \mathbf{q}$ proportional to $(\hat{\theta} \cdot \hat{k})^{2} \ll 1$.

The approximation used here is valid for small angular separations, $\kappa \gg 1$. Since the Ostriker-Vishniac effect produces anisotropies at multipole moments $\ell \geq 1000$, this flatsky approximation should lead to errors no greater than $O(0.1 \%)$. At large angular scales, we can also expect the anisotropy from the parallel term to be significant [10]. Also, the visibility function $g(w)$ should be smoothly varying over wavelengths at which there is significant power. Again, the visibility function for re-scattered CMB photons will be very broad, so this approximation should be fine for our calculation.

\footnotetext{
${ }^{1}$ We thank Wayne Hu for pointing out an error in this scaling in a previous draft.
} 
[1] M. Kamionkowski, D. N. Spergel, and N. Sugiyama, Astrophys. J. Lett. 426, L57 (1994).

[2] M. Tegmark, J. Silk, and A. Blanchard, Astrophys. J. 420, 484 (1994).

[3] Z. Haiman and A. Loeb, Astrophys. J. 483, 21 (1997).

[4] J. P. Ostriker and E. T. Vishniac, Astrophys. J., Lett. Ed. 306, L51 (1986).

[5] E. T. Vishniac, Astrophys. J. 322, 597 (1987).

[6] G. Efstathiou, in Large-Scale Motions, edited by V. Rubin and S. J. Coyne (Princeton University Press, Princeton, 1988).

[7] S. Dodelson and J. M. Jubas, Astrophys. J. 439, 503 (1995).

[8] W. Hu, D. Scott, and J. Silk, Phys. Rev. D 49, 648 (1994).

[9] F. M. Persi, D. N. Spergel, R. Cen and J. P. Ostriker, Astrophys. J. 442, 1 (1995).

[10] W. Hu and M. White, Astron. Astrophys. 315, 33 (1996).

[11] M. White, J. Carlstrom, and M. Dragovan, astro-ph/9712195.

[12] See the MAP homepage at http:// map.gsfc.nasa.gov, and the Planck homepage at http://astro.estec.esa.nl/SA-general/ Projects/Cobras/cobras.html

[13] G. Jungman, M. Kamionkowski, A. Kosowsky, and D. N. Spergel, Phys. Rev. D 54, 1332 (1996); J. R. Bond, G. Efstathiou, and M. Tegmark, astro-ph/9702100; M. Zaldarriaga, D. N. Spergel, and U. Seljak, astro-ph/9702157.
[14] See, e.g., P. J. E. Peebles, Principles of Physical Cosmology (Princeton University Press, Princeton, 1993).

[15] W. Hu and N. Sugiyama, Astrophys. J. 471, 542 (1996).

[16] J. M. Bardeen, J. R. Bond, N. Kaiser, and A. S. Szalay, Astrophys. J. 304, 15 (1986).

[17] E. F. Bunn and M. White, Astrophys. J. 480, 6 (1997).

[18] S. D. M. White, G. Efstathiou, and C. S. Frenk, Mon. Not. R. Astron. Soc. 262, 1023 (1993); P. T. P. Viana and A. Liddle, ibid. 281, 323 (1996).

[19] A. H. Jaffe and M. Kamionkowski (in preparation).

[20] D. J. Eisenstein, W. Hu, J. Silk, and A. Szalay, astro-ph/9710303.

[21] A. H. Jaffe, M. Kamionkowski, J. Silk, and N. Sugiyama (in preparation).

[22] See, e.g., R. Subrahmanyan R. D. Ekers, M. Sinclair, and J. Silk, Mon. Not. R. Astron. Soc. 263, 416 (1993), and references therein.

[23] G. Jungman, M. Kamionkowski, A. Kosowsky, and D. N. Spergel, Phys. Rev. Lett. 76, 1007 (1996).

[24] N. Kaiser, Astrophys. J. 388, 272 (1992).

[25] N. Kaiser, astro-ph/9610120.

[26] A. S. Monin and A. M. Yaglom, Statistical Fluid Mechanics (MIT Press, Cambridge, 1971), Vol. 2. 